\title{
Functional and Oncological Outcomes of Open Partial Laryngectomy vs. Transoral Laser Surgery in Supraglottic Larynx Cancer
}

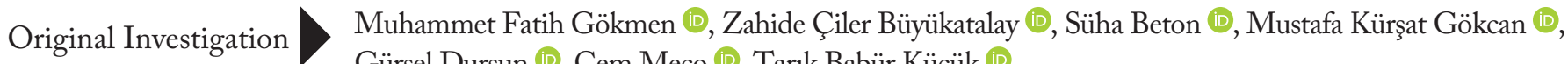 \\ Gürsel Dursun (D), Cem Meço (D), Tarık Babür Küçük (1) \\ Department of Otorhinolaryngology, Ankara University School of Medicine, Ankara, Turkey
}

\begin{abstract}
Objective: The aim of the presented study was to evaluate the outcomes of open partial laryngectomy (OPL) versus transoral laser surgery (TLS) in patients operated on for supraglottic laryngeal carcinoma based on functional parameters (duration of hospitalization, oral nutritional status and duration of transition to oral feeding, weaning status and duration after tracheotomy, and postoperative voice results) and oncological results (overall survival rate, disease-specific survival rate, recurrence, and presence of second primary tumors) in both groups.

Methods: All laryngeal carcinoma patients who had undergone either OPL or TLS in the period from January 2012 to March 2017 in our center and were followed-up at least for 36 months were included in the study. Statistical analyses were carried out using the t-test and the Mann-Whitney $U$ test to compare the means, and the Kaplan-Meier test for survival analysis.

Results: Fifty patients (44 males and 6 females) met the study criteria, of whom 31 had undergone OPL
\end{abstract}

and 19 TLS. Patients that underwent TLS had less tracheotomy needs, needed shorter hospitalization periods, and transitioned to oral feeding earlier, compared to those that underwent OPL. There were no significant differences between the two groups based on oral feeding rates and voice outcomes. The impact of TLS and OPL on organ preservation in supraglottic laryngeal cancer were comparable. For local recurrences, repeated endolaryngeal laser surgeries and adjuvant treatments could be used in the TLS patient group. There were no significant differences between the two groups based on overall survival rate and disease specific survival rate.

Conclusion: Although no significant differences were found in our study between the two surgical procedures in terms of oncological outcomes, TLS appeared to produce better functional outcomes in supraglottic laryngeal carcinoma than OPL.

Keywords: Larynx cancer, laryngectomy, laser therapy, tracheotomy, survival analysis
ORCID iDs of the authors: M.F.G. 0000-0002-4134-8375; Z.C..B. 0000-0002-0992-0079; S.B. 0000-0001-8195-4380; M.K.G. 0000-0002-4160-7349; G.D. 0000-0003-2542-8268; C.M. 0000-0001-8372-8045; T.B.K. 0000-0002-4781-2001.

Cite this article as: Gökmen MF, Büyükatalay ZÇ, Beton S, Gökcan MK, Dursun G, Meço C, et al. Functional and Oncological Outcomes of Open Partial Laryngectomy vs. Transoral Laser Surgery in Supraglottic Larynx Cancer. Turk Arch Otorhinolaryngol 2020; 58(4):227-33.

\section{Corresponding Author:}

Muhammet Fatih Gökmen; mfatihgokmen@gmail.com Received Date: 03.06 .2020 Accepted Date: 09.10.2020

Content of this journal is licensed under a Creative Commons Attribution 4.0 International License. Available online at www.turkarchotolaryngol.net

\section{Introduction}

Larynx is an important organ not only for speech but also for swallowing and breathing (1). These three are vital functions, and diseases of the larynx can lead to their impairment, and sometimes, total loss. As a result, most patients diagnosed with laryngeal cancer prefer treatment modalities that provide better functional results over those that provide the best oncological outcomes.

Presently, laryngeal cancers are more often diagnosed at earlier stages thanks to better access to healthcare and increased awareness about laryngeal cancer among patients and doctors. It is important to diagnose laryngeal cancers at an early stage because their treatment success is higher compared to other cancers. Conservative surgical intervention or radiotherapy improves survival rates and provides successful outcomes in preserving the functions (2).
While total laryngectomy is performed since the last quarter of the $19^{\text {th }}$ century, open partial laryngectomy (OPL) is used since the middle of the $20^{\text {th }}$ century with high oncological success $(3,4)$. Along with the advances in medical technologies, laser has been an important tool for medical practice. Laser surgery was first used in ophthalmology, then in other disciplines, and is successfully used in the treatment of laryngeal cancer since 1972 (5).

The aim of the presented study was to evaluate the oncological and functional outcomes in patients who underwent OPL or transoral laser surgery (TLS) for supraglottic laryngeal cancer at an academic tertiary referral center.

\section{Methods}

The study was approved by the institutional ethics committee of Ankara University School of Medicine (Approval Date: January 11, 2016; Approv- 
al Number: 01-12-16) and performed in accordance with the Declaration of Helsinki, using good clinical practice and by observing the regulatory requirements. Informed written consent was obtained from all participants or their legal representative before initiating any procedure.

The study was conducted at an academic tertiary referral center. All laryngeal carcinoma patients who underwent either OPL or TLS from January 2012 to March 2017 at our tertiary university hospital and were followed-up for at least 36 months were included in the study. Patients whose primary treatment was non-surgical, underwent total laryngectomy and radiotherapy, were initially treated for laryngeal cancer in other medical centers and then referred to our clinic, and patients who were followed-up for less than 36 months were excluded.

Detailed anamnesis, general systemic examinations, and otorhinolaryngological examinations of all patients were recorded. Smoking and alcohol consumption habits, as well as type and duration of major complaints were inquired. Videolaryngostroboscopy (VLS) was performed before the operation, and direct laryngoscopy was performed under general anesthesia in patients without a histopathological diagnosis. During direct laryngoscopy, the location of the tumor, its spread, relationship with surrounding tissues, and suitability for transoral laser surgery were evaluated.

At least one neck imaging such as computed tomography (CT), magnetic resonance imaging (MRI) or ultrasonography (USG) was performed preoperatively in all patients diagnosed with supraglottic cancer. All patients were scanned with either thorax CT or Positron Emission Tomography-Computed Tomography (PET/CT) for exclusion of secondary tumors/metastases to lungs.

All patients were informed about the surgical and non-surgical treatment modalities for laryngeal cancer, and detailed information about open surgery and transoral laser surgery were provided to patients who preferred surgical treatment. Temporary or permanent tracheostomy, infection, bleeding, and the possibility of total laryngectomy according to the condition of the tumor were explained to all patients who accepted the surgery, and in-

\section{Main Points}

- Transoral laser surgery provided shorter hospitalization times and earlier transitions to oral intake than open partial laryngectomy in supraglottic laryngeal cancer patients.

- There was no significant difference between the two groups based on oral feeding rates and weaning time from tracheotomy.

- Transoral laser surgery and open partial laryngectomy procedures had similar and acceptable oncological success rates.

- Although local recurrences were more frequently observed in the TLS group, this problem could be overcome with the help of repeated laser surgeries and radiotherapy, and a disease-free survival was ensured for patients. formed consent forms were obtained from all. Patients planned for TLS were also informed about the possibility of switching, if necessary, to open surgery during the operation, and asked to provide their signed informed consent forms for open surgery.

OPL and TLS were determined according their suitability for each patient. This was discussed with each patient. In patients who underwent OPL, supraglottic laryngectomy and selective neck dissection (level 2,3,4) were performed in the same session. Patients stayed at the postoperative intensive care unit (ICU) one night after the procedure. In patients who underwent TLS, first supraglottic laryngectomy was performed, and six weeks later, selective neck dissections (level 2, 3, and 4) were carried out in sequential order. TLS procedures were performed using a $\mathrm{CO}_{2}$ laser device (AcuPulse Duo, Lumenis, Yokneam, Israel) attached to an operation microscope (OPMI Lumera S7, Zeiss, Jena, Germany) with a micromanipulator. Following laryngectomy, biopsies were taken from the surgical margins before finishing the procedure and sent for frozen section analysis. In the post-operative six-week period, laryngectomy specimens were examined by pathologists, and, if necessary, a surgical margin extension procedure was performed under general anesthesia.

After the surgical procedure, functional outcomes, pathology results, follow-up periods, complications, and recurrences were retrospectively evaluated and carefully examined. Functional outcomes included hospitalization period, oral intake, time to oral intake after surgery, tracheotomy status, weaning time from tracheotomy, and voice quality. All frequencies could not be evaluated by the analysis software, and thereby an objective voice analysis could not be carried out because of the low frequency of patients'voices in the postoperative period. To evaluate the voice quality, patients were asked to assess their voice as good, moderate and poor, based on the adequacy and the impact on their daily communication skills. According to the recent TNM Classification of malignant tumors (TNM) system (American Joint Committee on Cancer [AJCC] 2017), clinical and pathological staging was determined following preoperative VLS, radiological examinations, intraoperative findings, and pathology results.

\section{Statistical Analysis}

IBM Statistical Package for the Social Sciences for Windows software v20.0 (IBM SPSS Corp.; Armonk, NY, USA) was used for statistical analyses. The OPL and TLS treatment groups were compared using the $t$ test for group means, and the Mann-Whitney U test for median values. Qualitative variables were evaluated with Pearson's chi-square test or Fisher's test. The Kaplan-Meier test was used for survival analysis. P values smaller than 0.05 were considered statistically significant.

\section{Results}

\section{Demographic Results}

A total of 50 patients (44 men [88\%] and 6 women [12\%]) with supraglottic laryngeal cancer who met the study criteria were included in the study. Male to female ratio was $7.34(44 / 6)$. 
Table 1. Sex, mean age and tumor location of the two treatment groups

\begin{tabular}{l|c|c}
\hline Sex & TLS & OPL \\
\hline Male & 18 & 26 \\
\hline Female & 1 & 5 \\
\hline Mean age (years) & 60.9 & 56.6 \\
\hline Tumor location & & \\
\hline Epiglottis & 11 & 19 \\
\hline Band ventricle (false vocal fold) & 7 & 9 \\
\hline Aryepiglottic fold & 1 & 2 \\
\hline Tongue base & 0 & 1 \\
\hline
\end{tabular}

TLS: transoral laser surgery; OPL: open partial laryngectomy

Table 2. Functional outcomes of the two treatment groups for the patients with supraglottic larynx cancer

\begin{tabular}{l|c|c|c}
\hline & TLS & OPL & $\mathrm{p}$ \\
\hline Hospitalization time (days) & 9.6 & 25.7 & 0.034 \\
\hline Oral feeding ratio (\%) & 69 & 71 & 0.17 \\
\hline Oral feeding duration (days) & 2.4 & 36.6 & 0.047 \\
\hline Intraoperative tracheotomy ratio (\%) & 16 & 100 & \\
\hline Weaning time from tracheotomy (days) & 70.5 & 44.7 & 0.68
\end{tabular}

TLS: transoral laser surgery; OPL: open partial laryngectomy

OPL was performed in 31 patients (62\%) and TLS in $19 \mathrm{pa}^{-}$ tients (38\%). Of those who underwent OPL 26 (83.9\%) were male and five (16.1\%) were female; and of those who underwent TLS 18 (94.7\%) were male and one (5.3\%) was female.

The mean age of the patients in the OPL group was 56.6 years (range: 43-83), and the mean age of those in the TLS group was 60.9 years (range: 41-79). There was no significant difference between the mean ages of the two groups. Age distributions were also similar in the two treatment groups.

Hoarseness was the most common complaint of the patients. Thirty-seven patients (74\%) consulted the doctor for hoarseness, eight patients (16\%) for swallowing difficulty and three patients $(6 \%)$ for throat pain. One patient (2\%) had breath shortness complaint and one patient (2\%) presented with a neck mass.

As for patients' histories: 47 (94\%) were smokers; and only three $(6 \%)$ who were diagnosed with laryngeal cancer were non-smokers. While nine patients (18\%) reported that they regularly consumed alcohol, others reported that they did not have this habit.
In terms of tumor localization, epiglottis was the leading site. While a majority of the tumors (in 30 patients, 60\%) were in the epiglottis, 10 patients (20\%) had the tumor in the left band ventricle, six patients (12\%) in the right band ventricle, three patients $(6 \%)$ on the aryepiglottic fold and one patient $(2 \%)$ on the base of the tongue (Table 1).

\section{Functional Outcomes}

The average length of hospital stays after TLS was $9.6 \pm 5.6$ days (range: $1-22$ ), and $25.7 \pm 10.1$ days (range: $13-51$ ) after open surgery. The difference was statistically significant $(\mathrm{p}=0.034)$.

Nine of the OPL (29\%) and six of the TLS patients (31\%) were discharged with a long-term feeding tube or percutaneous endoscopic gastrostomy (PEG) due to aspiration in oral feeding. The mean time to oral intake was $36.6 \pm 27.7$ days (range: 12 120 ) after open surgery and $2.4 \pm 3.0$ days (range: $0-9$ ) after TLS. While there was no statistical difference between the two groups in terms of the number of patients who could not switch to oral intake ( $\mathrm{p}=0.17)$, transition to oral intake was significantly better in the TLS group than in the open surgery group $(\mathrm{p}=0.047)$.

Intraoperative tracheotomy was performed in every patient who underwent open surgery. Twenty-three patients (74\%) were weaned from tracheotomy in an average of $44.7 \pm 46.5$ days (range: 14-180). Only three of the TLS patients (16\%) underwent intraoperative tracheotomy, and only one (5\%) was followed up with permanent tracheotomy. Of the two patients with temporary tracheotomy, tracheotomy was closed on the $39^{\text {th }}$ day in one patient, while the tracheotomy of the other $\mathrm{pa}^{-}$ tient was closed on the $102^{\text {nd }}$ postoperative day after the end of the chemoradiotherapy (CRT). There was less need for intraoperative and permanent tracheostomy in the TLS group, but the difference between the weaning times of the two groups was not significant $(\mathrm{p}=0.68)($ Table 2$)$.

Twenty-five patients in the open surgery group were evaluated subjectively in terms of voice. While two patients (8\%) defined their voice as poor, 13 patients evaluated as good (52\%) and 10 patients (40\%) as moderate. In the TLS group, 14 patients (74\%) considered their voice quality as good, four patients (21\%) as moderate, and one patient (5\%) as poor. Since the evaluation was performed subjectively, no statistical analysis was made.

\section{Oncological Outcomes}

All patients were evaluated clinically based on their preoperative examinations, imaging, and intraoperative findings (Table 3). Their pathological evaluations, on the other hand, were carried out after completion of the histopathologic examinations (Table 4). Updated AJCC classification of 2017 was used for staging (6).

The average follow-up period after surgery was 66 months (range: 36-99) in patients who underwent OPL and 62 months (range: 38-90) in patients who had TLS. There was no significant difference between the two groups in terms of follow-up periods. 
Table 3. Clinical $\mathrm{T}$ and $\mathrm{N}$ stages and percentages of the patients in the study

\begin{tabular}{l|c} 
Clinical staging & Number of patients (\%) \\
\hline $\mathrm{T} 1$ & $15(30)$ \\
\hline $\mathrm{T} 2$ & $31(62)$ \\
\hline $\mathrm{T} 3$ & $3(6)$ \\
\hline $\mathrm{T} 4$ & $1(2)$ \\
\hline $\mathrm{N} 0$ & $42(84)$ \\
\hline $\mathrm{N} 1$ & $2(4)$ \\
\hline $\mathrm{N} 2$ & $5(10)$ \\
\hline $\mathrm{N} 3$ & $1(2)$ \\
\hline Stage & \\
\hline 1 & $14(28)$ \\
\hline 2 & $25(50)$ \\
\hline 3 & $5(10)$ \\
\hline 4 & $6(12)$ \\
\hline Total & $50(100.0)$ \\
\hline
\end{tabular}

Table 4. Pathological $\mathrm{T}$ and $\mathrm{N}$ stages and percentages of the patients in the study

\begin{tabular}{l|c}
\hline Pathological staging & Number of patients (\%) \\
\hline T 1 & $17(34)$ \\
\hline T 2 & $24(48)$ \\
\hline T 3 & $5(10)$ \\
\hline T 4 & $4(8)$ \\
\hline N 0 & $39(78)$ \\
\hline N 1 & $5(10)$ \\
\hline N 2 & $5(10)$ \\
\hline N 3 & $1(2)$ \\
\hline Stage & \\
\hline 1 & $16(32)$ \\
\hline 2 & $19(38)$ \\
\hline 3 & $4(8)$ \\
\hline 4 & $11(22)$ \\
\hline Total & $50(100.0)$ \\
\hline
\end{tabular}

Eight patients (16\%) died during the follow-up period. Five had been treated with OPL. Three patients died due to regional neck involvement of tumor, one due to respiratory insufficiency as a result of lung malignancy and one due to cardiac arrest after a myocardial infarction. One OPL patient was admitted to our clinic with shortness of breath in the $15^{\text {th }}$ postoperative month, and total laryngectomy was performed as recurrence was de-

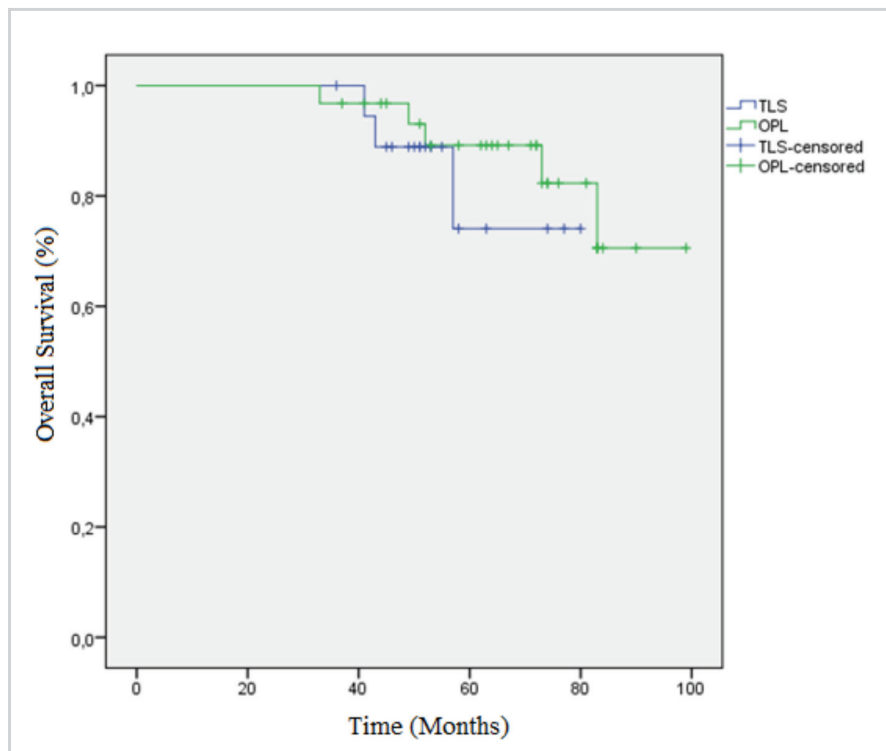

Figure 1. Overall survival rate for the TLS and OPL groups TLS: transoral laser surgery; OPL: open partial laryngectomy

tected. After total laryngectomy, follow-up continued without recurrence in the $41^{\text {st }}$ postoperative month. In the follow-up of patients who underwent OPL, secondary lung malignancy was detected in two patients and thyroid papillary carcinoma was observed in one patient. These three patients were surgically treated. In one patient who underwent OPL, biopsy was taken from a mass in the submental area in the $57^{\text {th }}$ postoperative month. This turned out to be squamous cell carcinoma. Surgery was recommended to the patient, but the patient wanted to have radiotherapy and was referred to the radiation oncology department. Overall, patients treated with OPL had a five-year survival rate of $83.9 \%$ and a five-year disease-free survival rate of $80.6 \%$.

Three patients in the TLS group died during the follow-up period. Two patients died due to regional recurrence of the tumor and one patient due to hepatic failure after chronic viral hepatitis. Local recurrence was detected in the control examination of five patients. These recurrences were detected in the $14^{\text {th }}, 18^{\text {th }}$, $24^{\text {th }}, 24^{\text {th }}$ and $30^{\text {th }}$ postoperative months, respectively. While four of these patients were treated with TLS again, one patient was referred to radiation oncology. Based on the most recent follow-ups, no local or regional progression was observed in the patients. The patients treated with TLS had a five-year survival rate of $84.2 \%$ and five-year disease-free survival rate of $79 \%$ (Figure 1).

\section{Discussion}

Laryngeal cancer is the second most common cancer in the head and neck region after skin cancer (7). In Turkey, laryngeal cancer is the eighth among the top ten most common cancers in men (8). Therefore, it is considered a major public health problem.

The treatment of laryngeal cancer has reached its present state by evolving through different modalities. Theodor Billroth was the first to describe total laryngectomy in 1873 (3). Radiother- 
apy started to be used for treatment in the late 1920s. Surgery modalities evolved from total laryngectomy to larynx preservation surgery. Suarez defined supraglottic horizontal laryngectomy (SHL) as a two-step surgery in 1944 , which was modified to its present version in 1960 (4). In 1975, Strong (5) reported the results of the first patients treated with laser surgery in Canada. Vaughan et al. (9) reported the first supraglottic laryngectomy with laser in 1978. The FDA approved transoral robotic surgery (TORS) for laryngeal cancer in 2009 (10).

TLS has become a reliable treatment option for supraglottic cancer in the recent years. Today, TLS represents a less invasive protocol that allows tumors to be removed with limited sacrifice of the normal tissue and by preserving the organ function (11). Functional outcomes of TLS are generally considered better than open surgery, and in many cases, they are comparable with radiotherapy and robotic surgery $(12,13)$. Other advantages of TLS include low morbidity and mortality, less need for tracheotomy and shorter duration of hospitalization (11-13). Furthermore, TLS has repeatedly demonstrated cure rates comparable to those of open surgery or primary radiotherapy in supraglottic cancer (11-13). Although it was previously common to believe that TLS was only an appropriate treatment in early stage (T1, T2) glottic and supraglottic cancers, the results shown by Ambrosch et al. (14) and Mannelli et al. (15) suggested that TLS is also a competent surgery in advanced stage $(\mathrm{T} 3, \mathrm{~T} 4)$ cancers.

The mean hospitalization time was 9.6 days after TLS and 25.7 days after open surgery in the present study. Kayhan et al. (16) performed TORS on 13 patients with supraglottic cancer and reported a mean hospitalization period of 15.4 days.

In the presented study, nine (29\%) OPL and six (31\%) TLS patients were discharged with long-term feeding tube or PEG due to aspiration in oral feeding. The mean duration of transition to oral intake was 36.6 days in open surgery and 2.4 days in TLS. Ambrosch et al. (14) reported that only $4 \%$ of their patients needed feeding tube or PEG one year after the operation. In their review study, van der Woerd et al. (17) evaluated a total of 640 supraglottic cancer patients in 10 papers, 320 of whom underwent surgery. For the management of aspiration, they reported a permanent tracheostomy or total laryngectomy rate of $2.6 \%$ and a permanent gastrostomy rate of $5.3 \%$. In our study swallowing rate at the time of discharge was somewhat lower compared to other studies. However, as a result of swallowing rehabilitation and patient adaptation, the patients did not need total laryngectomy or permanent tracheostomy for the purposes of aspiration control.

In the presented study, intraoperative tracheotomy was performed in all patients who underwent open surgery, and 23 patients (74\%) were decannulated at 44.7 days on the average. Only three (16\%) of the TLS patients underwent intraoperative tracheotomy, and only one patient (5\%) was followed up with permanent tracheostomy. In a study involving 91 patients evaluated over a period of ten years, Ambrosch et al. (14) reported that $13 \%$ of the patients required tracheotomy and $74 \%$ needed it in the early postoperative period. Estomba et al. (18) compared TLS and SHL groups, and reported that TLS patients required shorter hospitalization periods, faster decannulation times, and had less need for tracheotomy. Thus, the findings in our study are consistent with those of Estomba et al. (18).

Since vocal cords are preserved during organ protective surgeries in supraglottic cancers, voice quality does not change as much as it does in glottic tumors. The removal of false cords or arytenoids in expanded cases could considerably change the voice quality. Topaloğlu et al. (19) found a significant decrease in the maximum phonation time and the fundamental frequency of patients who underwent OPL compared to the control group. They did not, however, observe a significant difference in perceptual and subjective analysis. Roh et al. (20) stated that vocal functions, including the voice handicap index (VHI), the grade, roughness, breathiness, asthenia, strain (GRBAS) scale, and acoustic and aerodynamic parameters did not change significantly after TLS in supraglottic cancer. Oridate et al. (21) compared the voice related quality of life (VRQOL), VHI-10, and GRBAS scores of patients with T2 N0 supraglottic cancer against those of patients with T1a, T1b, and T2 N0 glottic cancers, and found no significant differences in functional outcomes. Since some of our patients continued their follow-up in other centers after five years, perceptual voice evaluations were not carried out on the phone, and only subjective analysis was performed in our study. Re-evaluation of the effects of patients' voices on their daily lives using surveys such as VRQOL and VHI would increase the accuracy of subjective analyses.

The clinical staging of patients was made based on preoperative examinations, imaging methods and intraoperative findings, while pathological staging was made based on the pathological examination of the larynx and neck dissection materials after surgical excision. In our study, the most important reason why advanced-stage patients were found to have such higher frequencies was deemed to be due to the classification of extracapsular involvement as N3b in the 2017 update of AJCC, and also the presence of lymph nodes in the bilateral neck even in the early stage of supraglottic tumors (6).

One of the most important oncological problems encountered during partial laryngectomy is the presence of tumors at the surgical margins. Surgical margin positivity results in higher recurrence rates, decreased local control, disease-specific survival, and lower overall survival rate. Therefore, it is recommended to take biopsies from the surgical margins for intraoperative frozen section analysis. Fang et al. (22) reported that cases with positive surgical margins mostly showed early local recurrence and poor prognosis in early stage glottic cancers. Similarly, Nakayama et al. (23) showed that positive surgical margins in open surgery led to early recurrence and increased mortality rates in 61 supracricoid laryngectomy cases.

In the presented study, the intraoperative frozen section analysis was performed in all TLS patients, and surgical margins were 
expanded in case of positive results. Nevertheless, postoperative positivity was detected in five patients in permanent section analysis, and local recurrence was observed during follow-up. Four of the recurrent tumors were re-excised with TLS and the other patient was treated with RT.

One of the most important factors determining survival in supraglottic cancers is the positivity of lymph nodes at the neck. Many studies confirmed that the probability of bilateral lymph node metastasis is high in supraglottic cancers. Ma et al. (24) found a total of $28.1 \%$ metastasis risk in clinical N0 necks, and this risk was $15.4 \%$ in T2, 32.5\% in T3, and 35.7\% in T4. Y11maz et al. (25) stated that the contralateral neck metastasis rate was $16 \%$ and the occult metastasis rate was $28 \%$ in a retrospective cohort study in Turkey. It was stated that whether the region where the tumor originates is far from or close to the epiglottic midline does not affect the metastasis rates. In contrast to these studies, Ferlito et al. (26) reported that only $1.6 \%$ of the supraglottic cancers had an involvement at level $2 \mathrm{~B}$ and $3.4 \%$ of them had a lymph node at level 4 in 272 clinical N0 patients. They suggested that the dissection of levels $2 \mathrm{~A}$ and 3 would be an adequate treatment. They also reported that bilateral neck dissection should be performed only in centrally located or bilateral tumors and that it would be appropriate to evaluate the other side of the neck in other situations.

In our study, selective neck dissections including levels 2, 3, 4 were performed bilaterally in 18 patients and unilaterally in 13 patients. In three OPL patients who had metastatic lymph nodes, the contralateral neck was operated in a separate session. No pathological lymph nodes were found in other ten patients. No tumors were detected in the contralateral necks of the 10 patients during follow-up. Of the 19 patients who underwent TLS, 12 had bilateral and seven had unilateral neck dissection. The contralateral neck was operated on in one patient due to a metastatic lymph node. No tumor was detected in the other side of the neck in the six patients during follow-up.

Ambrosch et al. (14) observed locoregional recurrence in $15.4 \%$ of their patients that underwent TLS. Five-year local control and five-year survival rates were $72 \%$ and $63 \%$, respectively. Karatzanis et al. (27) reported that overall five-year disease specific survival was $81.9 \%$ and local control was $90.8 \%$ in their series. While disease specific survival was $79.4 \%$ in $\mathrm{T} 1$ cases and $82.9 \%$ in T2 cases, local control was $87.0 \%$ in $\mathrm{T} 1$ cases and $92.3 \%$ in T2 cases. Karabulut et al. (28) found an overall survival rate of $88 \%$ in the TORS group and $95 \%$ in the OPL group. The authors reported the disease specific survival rate as $94 \%$ in the robotic surgery group and $95 \%$ in the open surgery group.

In our study, five patients in the OPL group died of various reasons. Patients had $83.9 \%$ overall survival and $80.6 \%$ disease-free survival rates. During the follow-up of the patients in the TLS group, local recurrence was observed in five patients. These recurrences were successfully treated with repeated laser surgery (four patients) and radiotherapy (one patient). Three patients in the TLS group died of various reasons. No local or region- al disease progression or recurrence was observed in the most recent follow-ups, and the overall survival rate was $84.2 \%$, and disease-free survival rate was $79 \%$.

\section{Conclusion}

The presented study showed that supraglottic cancer patients treated with TLS had shorter hospitalization times, earlier transition to oral intake, and less need for tracheotomy compared to the patients who underwent OPL. They also had better subjective voice quality outcomes. Even though local recurrences were more frequently observed in TLS compared to OPL, it was possible to protect the larynx and to ensure disease-free survival with the use of repeated laser surgeries and radiotherapy.

Ethics Committee Approval: Ethics committee approval was received for this study from the Ankara University School of Medicine (Aprproval Date: January 11, 2016; Approval Number: 01-12-16)

Informed Consent: Informed consent was obtained from patients or their legal representatives of the patients who participated in this study.

Peer-review: Externally peer-reviewed.

Author Contributions: Concept - T.B.K.; Design - M.F.G., S.B.; Supervision - Z.Ç.B., C.M.; Materials - M.K.G., G.D., T.B.K.; Data Collection and/or Processing - M.F.G., G.D.; Analysis and/or Interpretation - M.F.G.; Literature Search - M.F.G.; Writing - M.F.G., Z.Ç.B., S.B., C.M.; Critical Reviews - M.K.G.

Conflict of Interest: The authors have no conflicts of interest to declare.

Financial Disclosure: The authors declared that this study has received no financial support.

\section{References}

1. Armstrong WB, Vokes DE, Verma SP. Malignant tumors of the larynx. Flint PW, Haughey BH, Lund V, Niparko JK, Robbins KT, Thomas JR, et al, editors. Cummings Otolaryngology Head and Neck Surgery. 6th ed. Philadelphia: Elsevier Saunders; 2015. p.1601-33.

2. Weisman AR, Moe KS, Orloff LA. Neoplasms of the larynx and laryngopharynx. Snow JB, Ballenger JJ, editors. Ballenger's Otorhinolaryngology Head and Neck Surgery. 16th ed. Ontario: BC Decker; 2003. p.1255-97.

3. Billroth T, Gussenbauer C. Uber die erste durch Th. Billroth am Menschen Ausgeführte Kehlkopf Extirpation und die Anwendung eines kuntlichen Kelkopfes. Arch Klin Chir 1874; 17: 343-56.

4. Alonso JM. Conservative surgery of cancer of the larynx. Trans Am Acad Ophthalmol Otolaryngol 1947; 51: 633-42.

5. Strong, MS. Laser excision of carcinoma of the larynx. Laryngoscope 1975; 85: 1286-9.

6. Amin MB, Edge SB, Greene FL, Byrd DR, Brookland RK, Washington MK, et al, editors. AJCC Cancer Staging Manual. 8th ed. New York: Springer International Publishing; 2017.

7. Chu EA, Kim YJ. Laryngeal cancer: diagnosis and preoperative work-up. Otolaryngol Clin North Am 2008; 41: 673-95.

8. Bora Başara B, Soytutan Çağlar İ, Özdemir TA, Güler C, editors. Republic of Turkey Ministry of Health. Health Statistics Yearbook 2016. Ankara: Republic of Turkey Ministry of Health General Directorate of Health Research; 2017. p.36-40. 
9. Vaughan CW, Strong MS, Jako GJ. Laryngeal carcinoma: transoral treatment utilizing the CO2 laser. Am J Surg 1978; 136: 4903.

10. Iseli TA, Kulbersh BD, Iseli CE, Carroll WR, Rosenthal EL, Magnuson JS. Functional outcomes after transoral robotic surgery for head and neck cancer. Otolaryngol Head Neck Surg 2009; 141: 166-71.

11. Tufano RP, Stafford EM. Organ preservation surgery for laryngeal cancer. Otolaryngol Clin North Am 2008; 41: 741-55.

12. Iro H, Waldfahrer F, Altendorf-Hofmann A, Weidenbecher M, Sauer R, Steiner W. Transoral laser surgery of supraglottic cancer: follow-up of 141 patients. Arch Otolaryngol Head Neck Surg 1998; 124: 1245-50.

13. Agrawal A, Moon J, Davis RK, Sakr AW, Giri SPG, Valentino J, et al. Transoral carbon dioxide laser supraglottic laryngectomy and irradiation in stage I, II, and III squamous cell carcinoma of the supraglottic larynx: report of Southwest Oncology Group Phase 2 Trial S9709. Arch Otolaryngol Head Neck Surg 2007; 133: 104450.

14. Ambrosch P, Gonzalez-Donate M, Fazel A, Schmalz C, Hedderich J. Transoral laser microsurgery for supraglottic cancer. Front Oncol 2018; 8: 158.

15. Mannelli G, Lazio MS, Luparello P, Gallo O. Conservative treatment for advanced T3-T4 laryngeal cancer: meta-analysis of key oncological outcomes. Eur Arch Otorhinolaryngol 2018; 275: 2738.

16. Kayhan FT, Kaya KH, Altintas A, Sayin I. Transoral robotic supraglottic partial laryngectomy. J Craniofac Surg 2014; 25: 1422-6.

17. van der Woerd B, Patel KB, Nichols AC, Fung K, Yoo J, MacNeil SD. Functional outcomes in early (T1/T2) supraglottic cancer: a systematic review. J Otolaryngol Head Neck Surg 2018; 47: 76.

18. Estomba CMC, Reinoso FAB, Lorenzo AIL, Conde JLF, Nores JA, Hidalgo CS. Functional outcomes of supraglottic squamous cell carcinoma treated by transoral laser microsurgery compared with horizontal supraglottic laryngectomy in patients younger and older than 65 years. Acta Otorhinolaryngol Ital 2016; 36: 450-8.
19. Topaloğlu I, Salturk Z, Atar Y, Berkiten G, Büyükkoç O, Çakır O. Evaluation of voice quality after supraglottic laryngectomy. Otolaryngol Head Neck Surg 2014; 151: 1003-7.

20. Roh JL, Kim DH, Park CI. Voice, swallowing and quality of life in patients after transoral laser surgery for supraglottic carcinoma. J Surg Oncol 2008; 98: 184-9.

21. Oridate N, Homma A, Suzuki S, Nakamaru Y, Suzuki F, Hatakeyama $\mathrm{H}$, et al. Voice-related quality of life after treatment of laryngeal cancer. Arch Otolaryngol Head Neck Surg 2009; 135: 363-8.

22. Fang TJ, Courey MS, Liao CT, Yen TC, Li HY. Frozen margin analysis as a prognosis predictor in early glottic cancer by laser cordectomy. Laryngoscope 2013; 123: 1490-5.

23. Nakayama M, Okamoto M, Iwabuchi K, Mikami T, Seino Y. Clinical significance of intraoperative surgical margin study in supracricoid laryngectomy. Auris Nasus Larynx 2011; 38: 261-5.

24. Ma H, Lian M, Feng L, Li P, Hou L, Chen X, et al. Factors contributing to lymph node occult metastasis in supraglottic laryngeal carcinoma cT2-T4 N0M0 and metastasis predictive equation. Chin J Cancer Res 2014; 26: 685-91.

25. Yılmaz T, Süslü N, Atay G, Günaydın RÖ, Bajin MD, Özer S. The effect of midline crossing of lateral supraglottic cancer on contralateral cervical lymph node metastasis. Acta Otolaryngol 2015; 135: 484-8.

26. Ferlito A, Silver CE, Rinaldo A. Selective neck dissection (IIA, III): a rational replacement for complete functional neck dissection in patients with N0 supraglottic and glottic squamous carcinoma. Laryngoscope 2008; 118: 676-9.

27. Karatzanis AD, Psychogios G, Zenk J, Waldfahrer F, Hornung J, Velegrakis GA, et al. Evaluation of available surgical management options for early supraglottic cancer. Head Neck 2010; 32: 104855.

28. Karabulut B, Deveci I, Sürmeli M, Şahin-Yilmaz A, Oysu Ç. Comparison of functional and oncological treatment outcomes after transoral robotic surgery and open surgery for supraglottic laryngeal cancer. J Laryngol Otol 2018; 132: 832-6. 